\title{
Isolement et activité nitrogénasique de Burkholderia vietnamiensis, bactérie fixatrice d'azote associée au riz (Oryza sativa L) cultivé sur un sol sulfaté du Viêt-nam
}

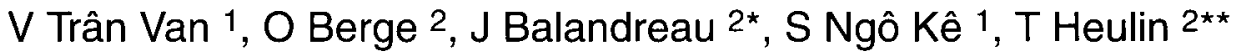 \\ 1 Institute of Tropical Biology (ITB), Vietnam National Center for Natural Sciences and Technology (VNCNST), \\ 1 Mac Dinh Chi Street, Ho Chi Minh Ville, Viêt-nam; \\ 2 Laboratoire d'écologie microbienne de la rhizosphère, Centre de pédologie biologique, UPR 6831 du CNRS associée \\ à l'université Henri-Poincaré Nancy-I, BP 5, F-54501, Vandœuvre-lès-Nancy cedex, France
}

(Reçu le 29 février 1996; accepté le 13 septembre 1996)

\begin{abstract}
Summary - Isolation and nitrogenase activity of Burkholderia vietnamiensis, a nitrogen-fixing bacterium associated with rice (Oryza sativa $L$ ) on a sulphate acid soil of Vietnam. To study the dominant diazotrophs associated with rice in Vietnam, young rice plants were grown on a Vietnamese sulphate acid soil in a phytotron; under these conditions, a maximum nitrogenase activity (estimated with acetylene reduction activity technique) of ca 360 $\mathrm{nmol} \mathrm{C}_{2} \mathrm{H}_{4} \mathrm{~h}^{-1}$ plant ${ }^{-1}$, was measured on day 8. At this stage, $\mathrm{N}_{2}$-fixing bacteria were counted and isolated, using the 'spermosphere model' technique, in which exudates of an aseptically grown rice plantlet are the only $C$ source for bacteria. The contribution of $\mathrm{N}_{2}$-fixing bacteria to the microflora (total bacteria, $6.5 \times 10^{8}$ colony forming units $g^{-1}$ soil) was shown to be close to $40 \%$ in the initial rhizosphere soil. Twelve representative isolates were further studied and proved to be Burkholderia vietnamiensis. They exhibited a low $\mathrm{pH}$-adapted nitrogenase activity. Four of them were selected for comparison with six other strains of Azospirillum lipoferum, A brasilense and Enterobacter cloacae for their nitrogenase activities in the presence of axenically grown rice plantlets. Strain TVV75 of B vietnamiensis was more efficient (1 $900 \mathrm{nmol} \mathrm{C}_{2} \mathrm{H}_{4}$ tube-1 day-1) than the others, with the exception of strain MRB16 of A lipoferum (from Bangladesh). Consequently, strain TVV75 of B vietnamiensis has been retained for subsequent inoculation trials in Vietnam where yield increases were obtained.
\end{abstract}

rhizosphere / nitrogen fixation / Oryza sativa $L=$ rice / dominant microflora / spermosphere model / acid sulphate soil / Burkholderia vietnamiensis / Vietnam

Résumé - L'étude de la microflore bactérienne fixatrice d'azote dominante associée au riz a été menée sur un sol sulfaté acide du Viêt-Nam. Une première expérience a montré que l'activité nitrogénasique (estimée par mesure de l'activité réductrice d'acétylène) du système sol-plante intact était maximale après huit jours de croissance, en chambre climatisée (360 nmoles de $\mathrm{C}_{2} \mathrm{H}_{4} \mathrm{~h}^{-1}$ plante- ${ }^{-1}$ ). Dans une deuxième expérience, le comptage et l'isolement

* Adresse actuelle : Laboratoire d'écologie microbienne du sol, UMR 5557 du CNRS, université Claude-Bernard, 43, boulevard du 11Novembre-1918, F-69622 Villeurbanne, France

** Correspondance et tirés à part : Lemir-DEVM, UMR 163 CNRS-CEA, CEA Cadarache, F-13108, Saint-Paul-lez-Durance cedex, France 
des bactéries fixatrices d'azote les plus abondantes de la rhizosphère après 8 jours de culture, ont été réalisés à l'aide du modèle spermosphère. Dans ce système les exsudats d'une plante axénique de riz constituent l'unique source de carbone pour la croissance des bactéries. Le peuplement des bactéries fixatrices d'azote a été évalué dans le sol de départ à $40 \%$ de la microflore totale cultivable qui atteint $6,510^{8}$ UFC (unités formant colonies) par gramme de sol sec. Douze souches fixatrices parmi les plus abondantes ont été particulièrement étudiées. Ces souches appartiennent à la nouvelle espèce Burkholderia vietnamiensis. Elles ont une fixation d'azote adaptée aux bas $\mathrm{pH}$. Le modèle spermosphère a été utilisé pour comparer l'activité nitrogénasique de quatre isolats de $B$ vietnamiensis et de six souches de collection appartenant aux genres Azospirillum et Enterobacter, isolées d'autres sols. La souche $B$ vietnamiensis TVV75 s'est avérée plus efficiente que toutes les autres souches testées (1900 nmoles de $\mathrm{C}_{2} \mathrm{H}_{4}$ tube $^{-1}$ jour $\left.{ }^{-1}\right)$ exception faite de la souche MRB16 d'A lipoferum (isolée au Bangladesh). La souche TVV75 de $B$ vietnamiensis avait été retenue sur ce critère pour inoculer le riz au Viêt-nam dans des essais montrant des augmentations de récolte.

rhizosphère / fixation d'azote / Oryza sativa $L=$ riz / sol sulfaté acide / modèle spermosphère / Burkholderia vietnamiensis / Viêt-nam

\section{INTRODUCTION}

Des études de bilan d'azote (Watanabe et Lee, 1977 ; App et al, 1986 ; Wetselaar, 1981) ont démontré depuis longtemps l'importance du rôle des microorganismes fixateurs d'azote en riziculture irriguée traditionnelle. La fixation d'azote peut y être élevée (Roger et Ladha, 1992) et représenter une proportion non négligeable des besoins en azote de la plante. Plusieurs types de microorganismes sont impliqués et, parmi eux, les bactéries fixatrices d'azote hétérotrophes de la rhizosphère du riz. En effet, les diazotrophes sont extrêmement abondants à la fois en surface et à l'intérieur des racines du riz (Ladha et al, 1987 ; Omar et al, 1989 ; Rahman et al, 1996 ; Roussos et al, 1980 ; Thomas-Bauzon et al, 1982 ) et peuvent représenter jusqu'à $80 \%$ de la microflore totale (Watanabe et Barraquio, 1979 ; Barraquio et Watanabe, 1981).

Plus que leur abondance, c'est la diversité des fixateurs d'azote associés à la rhizosphère du riz qui constitue le caractère le plus original de cette niche écologique très particulière; du point de vue taxonomique, tous les grands groupes d'eubactéries y ont été reconnus, qu'il s'agisse de firmicutes tels que divers Clostridium (Chistyakova et Kalininskaya, 1984), ou Bacillus (Rasolomanana et Balandreau, 1987 ; Khammas et Kaiser, 1992), de protéobactéries $\alpha$ telles qu'Azospirillum (Lakshmi Kumari et al, 1976 ; Khammas et al, 1989), Beijerinckia (Starkey et De, 1939), ou Sphingomonas paucimobilis (Bally et al, 1990), de protéobactéries $\beta$ telles qu'Alcaligenes faecalis (Yuansheng et al, 1981), Herbaspirillum seropedicae (Baldani et al, 1986), et de protéobactéries $\gamma$, telles qu'Enterobacter cloacae (Bally et al, 1983), 'Enterobacter' agglomerans et Kelbsiella planticola (Yoo et al, 1986;
Omar et al, 1989) ou Klebsiella oxytoca (Bally et al, 1983). Par amplification directe de nifH, suivie d'un clonage et de nombreux séquençages, Ueda et al (1995) ont pu confirmer cette diversité, et même montrer que de nombreux microorganismes porteurs de gènes de la nitrogénase restaient à décrire dans la rhizosphère du riz.

Au Viêt-nam, la microflore fixatrice d'azote des sols de riziculture est très peu connue (Duong Duc et al, 1987). Son utilisation pour augmenter la production constitue pourtant une alternative intéressante par rapport à l'emploi d'engrais azotés, et a fait l'objet d'un projet de coopération financé dans le cadre STD $2 \mathrm{~A}$ de la Communauté économique européenne.

La première étape du projet consistait à mesurer l'activité et à décrire la composition de la microflore diazotrophe, dans un sol de type sulfaté acide, fréquent au sud du Viêt-nam. On a choisi pour cette étude la méthode du modèle spermosphère, déjà utilisée pour le riz par Omar et al (1989) et Rahman et al (1996), pour le blé par Heulin et al (1994) et pour le maïs par Berge et al (1991).

Une deuxième étape a consisté à comparer les souches entre elles pour leur activité nitrogénasique en présence de la plante. On a choisi pour cela le protocole proposé en 1989 par Heulin et al, qui permet la comparaison de nombreuses souches en modèle spermosphère.

Dans une troisième étape, dont les résultats ont déjà été publiés, des expériences en pots ont permis de vérifier que l'inoculation des souches sélectionnées avait un effet positif sur la croissance du riz et sa production (Trân Van et al, 1994). L'objectif ultime était de réaliser des expériences d'inoculation au champ, dont les résultats sont en cours de publication. 


\section{MATÉRIEL ET MÉTHODES}

\section{Isolement des bactéries fixatrices d'azote les plus abondantes de la rhizosphère du riz}

La méthodologie qui a été suivie pour l'isolement des bactéries fixatrices d'azote de la rhizosphère, est celle décrite par Omar et al (1989).

\section{Le sol}

Le sol a été prélevé dans l'arrondissement de Bình Thanh sur le terrain de la station expérimentale de la coopérative Bình Quoi, située à $10 \mathrm{~km}$ au nord-est de Hô Chi Minh Ville, au sud du Viêt-nam. Cette exploitation agricole pratique une rotation semestrielle riz/jachère. Lors du prélèvement de l'échantillon, le sol portait une culture de riz au début de la floraison. Ce sol est sulfaté acide (Sali-sulfi-Thionic Fluvisols selon la classification $\mathrm{FAO}$ ) avec une texture argilolimoneuse ; ses caractéristiques physicochimiques sont présentées dans le tableau I. Les prélèvements ont été stockés dans des sacs en plastique à l'obscurité à $4{ }^{\circ} \mathrm{C}$ jusqu'à l'utilisation.

\section{Culture du riz au phytotron}

Le riz, cv Huyêt Ròng, a été cultivé sur le sol de Bình Thanh, en chambre de culture dans les conditions suivantes : les graines calibrées, stérilisées et prégermées ( 3 jours), ont été placées individuellement dans des tubes en verre contenant $35 \mathrm{~g}$ de sol (matière sèche) préalablement submergé. Ces tubes étaient enveloppés à leur base par une feuille d'aluminium pour maintenir le sol et les racines à l'obscurité. La température et l'humidité relatives furent maintenues respectivement à $30{ }^{\circ} \mathrm{C}$ et $90 \%$ pendant le jour (photopériode 13 heures) et $24{ }^{\circ} \mathrm{C}$ et $70 \%$, la nuit. Lors de la première expérience, l'activité nitrogénasique des systèmes sol-plantes intacts a été suivie quotidiennement entre le $3^{e}$ et le $10^{e}$ jour de culture sur les mêmes tubes, par la méthode d'incubation sous acétylène. Au moment de la mesure, les systèmes étaient incubés sous $10 \%$ d'acétylène pendant trois heures et des prélèvements d'échantillons gazeux effectués régulièrement pour suivre le dégagement d'éthylène par chromatographie en phase gazeuse. Dans une deuxième expérience la culture du riz, en quatre répétitions, a été menée jusqu'au huitième jour, date à laquelle l'activité nitrogénasique déterminée dans la première expérience était maximale. L'isolement fut effectué le huitième jour à partir du sol rhizosphérique.

\section{Isolement et purification des souches}

Le sol rhizosphérique est défini ici comme le sol adhérant à la racine. L'isolement a donc été conduit à partir d'un broyat des racines et du sol y adhérant. Le sol rhizosphérique des quatre plantes de la deuxième expérience constituait le matériel de départ de l'isolement.
Des suspensions-dilutions de ce broyat furent inoculées à des tubes contenant une plante de riz axénique, en germination sur un milieu minéral WAT semi-solide ( $0,5 \%$ d'agar) de composition suivante :

- solution $\mathrm{A}: \mathrm{H}_{3} \mathrm{BO}_{3} 0,75 \mathrm{~g}, \mathrm{ZnSO}_{4} 7 \mathrm{H}_{2} \mathrm{O} 0,55 \mathrm{~g}$, $\mathrm{CoSO}_{4} 7 \mathrm{H}_{2} \mathrm{O} 0,35 \mathrm{~g}, \mathrm{CuSO}_{4} 4 \mathrm{H}_{2} \mathrm{O} 0,0218 \mathrm{~g}, \mathrm{MnCl}_{2}$ $4 \mathrm{H}_{2} \mathrm{O} 0,020 \mathrm{~g}$, eau $1000 \mathrm{~mL}$;

- solution B : $\mathrm{FeSO}_{4} 7 \mathrm{H}_{2} \mathrm{O} 0,8 \mathrm{~g}, \mathrm{MgSO}_{4} 7 \mathrm{H}_{2} \mathrm{O} 4,0 \mathrm{~g}$, $\mathrm{Na}_{2} \mathrm{MoO}_{4} 2 \mathrm{H}_{2} \mathrm{O} 0,118 \mathrm{~g}, \mathrm{CaCl}_{2} 2 \mathrm{H}_{2} \mathrm{O} 4,0 \mathrm{~g}$, EDTA $0,8 \mathrm{~g}$, solution $\mathrm{A} 4 \mathrm{~mL}$, eau $1000 \mathrm{~mL}$;

- solution finale : $\mathrm{KH}_{2} \mathrm{PO}_{4} 1,8 \mathrm{~g}, \mathrm{~K}_{2} \mathrm{HPO}_{4} 2,7 \mathrm{~g}$, solution $\mathrm{B} 50 \mathrm{~mL}$, eau $1000 \mathrm{~mL}, \mathrm{pH} \mathrm{6,8}$, autoclavage 30 minutes à $110^{\circ} \mathrm{C}$

Les tubes ont été fermés hermétiquement et incubés sous $1 \%$ d'acétylène suivant la méthodologie du modèle spermosphère décrite par Omar et al (1989). Au cours de cette étape, le nombre de fixateurs d'azote a été déterminé par la méthode du nombre le plus probable (MPN), en considérant le nombre et la répartition des tubes ayant formé de l'éthylène en deux jours à $30^{\circ} \mathrm{C}$. Le nombre de bactéries totales cultivables a été également estimé par étalement des dilutions du broyat de sol rhizosphérique sur milieu gélosé nutritif (NA Difco).

Les tubes inoculés par les dilutions les plus fortes et montrant encore une activité nitrogénasique ont été sélectionnés pour l'étape suivante ; leur contenu (milieu + racines), broyé et dilué est réparti par étalement en surface d'une gélose (15 g/L) WAT 4C contenant le milieu WAT additionné de quatre sources de carbone (glucose 5,0 g/L, mannitol $5,0 \mathrm{~g} / \mathrm{L}$, amidon $4,5 \mathrm{~g} / \mathrm{L}$, acide malique $3,5 \mathrm{~g} / \mathrm{L}$ ) et dont le $\mathrm{pH}$ est ajusté à 6,8 . Les colonies isolées ont été prélevées et purifiées et l'activité nitrogénasique de chaque isolat ainsi obtenu a été testée individuellement sur milieu WAT $4 C$.

\section{Comparaison de l'activité nitrogénasique des bactéries fixatrices d'azote de la rhizosphère du riz}

\section{Les souches bactériennes}

L'ensemble des souches utilisées dans cette étude figure dans le tableau II. En plus des souches de $B$ vietnamiensis isolées du sol vietnamien figurent des souches de collection isolées de la rhizosphère du riz avec la même méthodologie, dans diverses régions du monde.

\section{Activité nitrogénasique in vitro}

L'activité nitrogénasique, dans diverses conditions d'acidité, des souches $B$ vietnamiensis TVV70 et Azospirillum irakense KBC1 a été mesurée sur milieu RCV semi-solide (Heulin et al, 1987) modifié par addition de quatre sources de carbone (glucose $5,0 \mathrm{~g} / \mathrm{L}$, mannitol $5,0 \mathrm{~g} / \mathrm{L}$, amidon $4,5 \mathrm{~g} / \mathrm{L}$, acide malique $3,5 \mathrm{~g} / \mathrm{L}$ ) et ajusté à $\mathrm{pH} 4,5,6$ et 7 . Les bactéries en 


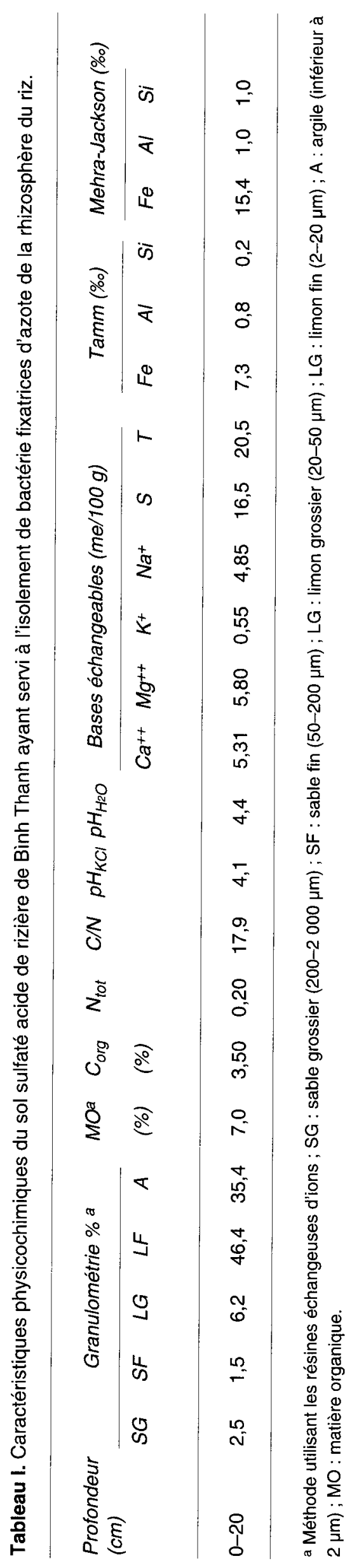


Tableau II. Origine des souches bactériennes utilisées dans l'étude comparative des activités nitrogénasiques, après inoculation de plantule de riz en modèle spermosphère.

\begin{tabular}{|c|c|c|c|}
\hline Souches & Origine & Source & Références \\
\hline \multicolumn{4}{|l|}{ Burkholderia vietnamiensis } \\
\hline TVV69, TVV70, TVV71, TVV75'T (LMG 10929') & Viêt-nam & Lemir & Gillis et al (1995) \\
\hline \multicolumn{4}{|l|}{ Azospirillum lipoferum } \\
\hline$-4 B$ & France & Lemir & Bally et al (1983) \\
\hline - MRB16 & Bangladesh & Lemir & Rahman et al (1996) \\
\hline \multicolumn{4}{|l|}{ Azospirillum brasilense } \\
\hline$-\mathrm{NO} 40$ & Égypte & Lemir & Omar et al (1989) \\
\hline - ATCC $29145^{\top}(\mathrm{Sp} 7)$ & Brésil & ATCC & Tarrand et al (1978) \\
\hline \multicolumn{4}{|l|}{ Azospirillum irakense } \\
\hline$-\mathrm{KBC}_{1}^{\top}\left(\mathrm{CIP} 10311^{\top}\right)$ & Irak & Cip & Khammas et al (1989) \\
\hline \multicolumn{4}{|l|}{ Enterobacter cloacae } \\
\hline- NO13 & Égypte & Lemir & Omar et al (1989) \\
\hline
\end{tabular}

T : souche type de l'espèce. ATCC : American Type Culture Collection, 12301 Parklawn Drive, Rockville MD 20852 (États-Unis). LMG : Culture Collection, Laboratorium voor Microbiologie, université de Gand, Gand, Belgique. Cip : Collection de l'Institut Pasteur, Paris, France. Lemir : Laboratoire d'écologie microbienne de la rhizosphère, CPB, CNRS Vandœuvre, France.

phase exponentielle de croissance ont été lavées puis inoculées (huit répétitions) à une densité de $210^{7}$ bactéries par tube et incubées sous $10 \%$ d'acétylène à $30{ }^{\circ} \mathrm{C}$. Les dosages d'éthylène ont été effectués tous les deux jours pendant sept jours et la vitesse maximale de dégagement d'éthylène $\left(R_{\max } \ln\right.$ nmoles $\mathrm{C}_{2} \mathrm{H} 4$ jour $^{-1}$ tube $^{-1}$ ) a été calculée.

\section{Activité nitrogénasique in planta}

La technique du modèle spermosphère a été utilisée pour mesurer et comparer l'activité nitrogénasique des souches bactériennes isolées de la rhizosphère en association avec le riz (Heulin et al, 1989). Des plantes de riz axéniques furent inoculées par des cultures pures ( $310^{7}$ bactéries tube ${ }^{-1}$ ) et incubées sous $10 \%$ d'acétylène à l'obscurité à $30^{\circ} \mathrm{C}$. L'éthylène a été dosé après $1,3,5$ et 7 jours d'incubation et la vitesse maximale de dégagement d'éthylène $\left(R_{\max }\right)$ calculées. $R_{\text {max }}$ a été retenue comme mesure de l'efficience des souches en accord avec Heulin et al, 1989. Deux expériences indépendantes dans lesquelles toutes les souches ont été testées (cinq répétitions par souche) furent réalisées.

\section{Traitement statistique des données}

Le traitement statistique des données a été effectué par analyse de variance à deux facteurs et leur interaction avec Statgraphics (Version 5.0, Uniware STSC Inc, États-Unis).

\section{RÉSULTATS}

\section{Activité réductrice d'acétylène (ARA) des systèmes sol-plante avant isolement}

L'activité réductrice d'acétylène globale mesurée sur un dispositif complet comprenant du riz mis en culture sur le sol sulfaté acide a été comparée à celle d'un témoin non planté et placé dans les mêmes conditions. La figure 1 montre que l'effet de la plante s'observe dès le troisième jour. De plus, l'activité nitrogénasique des systèmes plantés qui était de 20 nmoles de $\mathrm{C}_{2} \mathrm{H}_{4} \mathrm{~h}^{-1}$ plante-1 au troisième jour, forme un pic, au huitième jour, atteignant 360 nmoles de $\mathrm{C}_{2} \mathrm{H}_{4} \mathrm{~h}^{-1}$ plante ${ }^{-1}$, avant de décroître progressivement. Cette évolution de l'activité réductrice d'acétylène est tout à fait comparable à celle obtenue précédemment. dans une étude similaire sur du riz et des sols égyptiens par Omar et al (1989). L'exiguïté des tubes utilisés pour la culture du riz, ainsi que l'épuisement des réserves de la semence limitent la fixation d'azote à partir du neuvième jour et c'est pourquoi les isolements ont toujours été réalisés au huitième jour de culture.

Lors d'une nouvelle expérience, après huit jours de culture le sol rhizosphérique de quatre plantes a été prélevé pour en isoler les microor- 
ganismes responsables de l'activité nitrogénasique.

\section{Taille du peuplement fixateur d'azote}

La taille du peuplement des fixateurs d'azote dans la rhizosphère du riz de 8 jours a été estimée par la méthode des MPN à $2,8 \times 10^{8}$ bactéries fixatrices d'azote par gramme de sol rhizosphérique (matière sèche). Au même moment, la microflore totale cultivable sur gélose nutritive, était de $6,5 \times 10^{8}$ bactéries par $\mathrm{g}$ de sol rhizosphérique (matière sèche). Le peuplement de fixateurs d'azote représente environ $40 \%$ de la microflore totale cultivable dans ces systèmes. Ce pourcentage est remarquablement élevé, et a facilité l'isolement ultérieur des souches bactériennes fixatrices d'azote dominantes.

\section{Isolement des bactéries fixatrices d'azote les plus nombreuses}

Les tubes, contenant chacun une plante de riz axénique inoculée par une dilution du broyat de sol rhizosphérique, ont été incubés sous acétylène. Après une semaine d'incubation, les cinq tubes inoculés par la dilution $10^{-7}$ avaient tous produit de l'éthylène alors qu'un seul des cinq Rmax

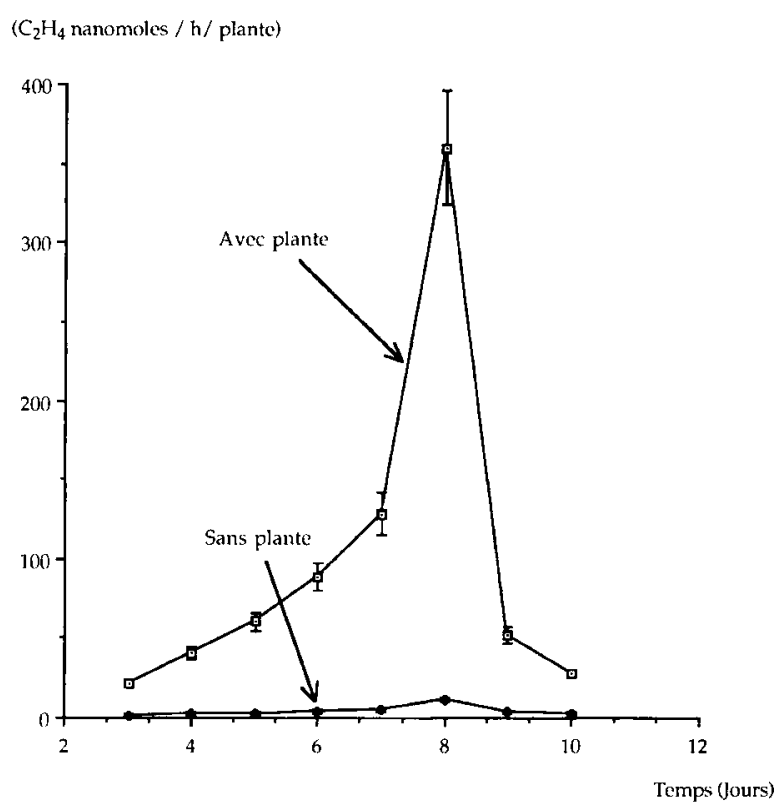

Fig 1. Évolution de l'activité réductrice d'acétylène d'un système complet sol-plante. Cas du riv cv Huyêt Ròng, cultivé sur le sol de Bình Thanh au phytotron. Au moment de la mesure les systèmes sont incubés sous $\mathrm{C}_{2} \mathrm{H}_{2}$ pendant 3 heures et le dégagement de $\mathrm{C}_{2} \mathrm{H}_{4}$ est suivi par chromatographie en phase gazeuse. Chaque point représente la moyenne \pm l'intervalle de confiance $(90 \%)$. tubes inoculés par la dilution $10^{-8}$ était positif pour ce test. Ce tube contenait donc les bactéries fixatrices d'azote les plus abondantes dans la rhizosphère de départ et a été sélectionné pour l'isolement ultérieur.

Après purification, un total de 82 souches pures a été obtenu à partir de ce tube. Ces souches étaient toutes positives pour le test de réduction d'acétylène sur milieu WAT $4 \mathrm{C}$ et 12 d'entre elles ont montré une activité particulièrement élevée par rapport aux 70 autres souches. Ces 12 souches de $B$ vietnamiensis ont été retenues pour la suite du travail.

\section{Effet du pH sur l'activité réductrice d'acétylène (ARA) de $B$ vietnamiensis}

L'activité nitrogénasique des souches TVV70 et $\mathrm{KBC} 1$ a fortement varié en fonction du $\mathrm{pH}$, ce qui est confirmé par les résultats de l'analyse de variance présentés dans le tableau III : l'effet du $\mathrm{pH}$ sur $\mathrm{R}_{\max }$ est significatif. Sur la figure 2 est représenté l'Ara des souches, TVV70 et KBC1. $\mathrm{R}_{\text {max }}$ était très faible à $\mathrm{pH} 4 \quad(<500$

$\operatorname{Rmax}\left(\mathrm{C}_{2} \mathrm{H}_{4} /\right.$ tube/jour $)$

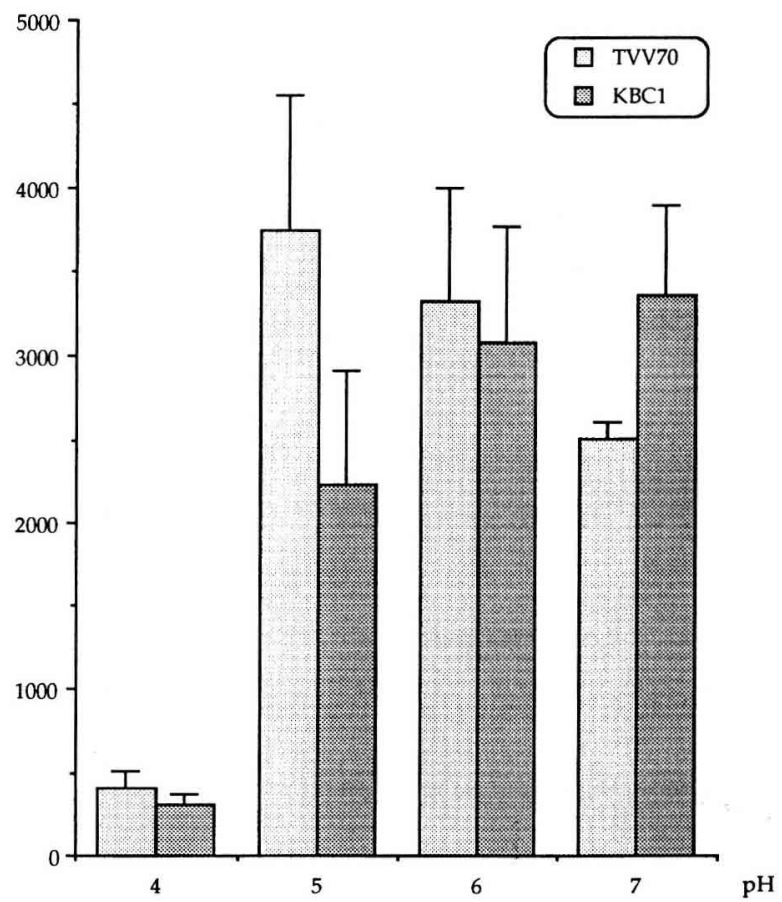

Fig 2. Effet du pH sur l'activité réductrice d'acétylène de Burkholderia vietnamiensis TVV70 isolée d'un sol sulfaté acide vietnamien et d'Azospirillum irakense $\mathrm{KBC} 1$ isolée d'un sol irakien. Les souches pures ont été inoculées in vitro en milieu semi-solide et incubés sous $10 \%$ de $\mathrm{C}_{2} \mathrm{H}_{2}$. Chaque barre d'histogramme représente la moyenne ( \pm l'intervalle de confiance à $95 \%$ ) de 7 répétitions à $\mathrm{pH} 4$ et 5 et de huit répétitions à $\mathrm{pH} 6$ et 7 
Tableau III. Résultats de l'analyse de variance de $\mathrm{R}_{\max } \mathrm{nmol} \mathrm{C}_{2} \mathrm{H}_{4}$ /tube/jour) en fonction de la souche inoculée (Burkholderia vietnamiensis TVV70 et Azospirillum irakense KBC1) et du pH du milieu.

\begin{tabular}{llllll} 
Source de variations & $D D L$ & Somme des carrés & Carrés moyens & $F$ & $P$ \\
\hline Bactérie & & & & & \\
pH & 1 & 971634 & 971634 & 2,513 & 0,12 \\
Bactérie $\times \mathrm{pH}$ & 3 & 78139018 & 26046339 & 67,37 & $<0,0000$ \\
Résidu & 3 & 10412604 & 3470868 & 8,98 & 0,0001 \\
Total & 52 & 20102541 & 386587 &
\end{tabular}

Les tests $\mathrm{F}$ font intervenir le rapport entre le carré moyen des écarts du facteur étudié et le résidu.

nmoles/tube/jour) alors que, pour des $\mathrm{pH}$ compris entre 5 et 7 , il était beaucoup plus élevé ( $>2000$ nmoles/tube/jour). L'effet souche n'est pas significatif, c'est-à-dire que, toutes valeurs de $\mathrm{pH}$ confondues, les deux souches ont des activités comparables alors que l'interaction entre le $\mathrm{pH}$ et la souche a un effet significatif (tableau III). L'écart entre les activités des deux bactéries est surtout net à $\mathrm{pH} 5$ où TVV70 a une activité maximale qui décroît ensuite avec le pH alors qu'à ce $\mathrm{pH} \mathrm{KBC1}$ a une activité plus faible qui augmente ensuite avec le $\mathrm{pH}$.

\section{Aptitude des souches de B vietnamiensis, à fixer l'azote en présence de la plante et comparaison avec d'autres souches isolées de la rhizosphère du riz}

L'utilisation du modèle spermosphère a permis d'estimer l'efficacité de la fixation de l'azote des isolats, en conditions gnotobiotiques, en présence de la plante. Elle a permis de les comparer non seulement entre elles, mais également avec d'autres souches isolées de la rhizosphère du riz en utilisant la procédure décrite par Heulin et al (1989). Nous avons choisi pour cela des souches efficientes d'origines diverses (tableau II). Nous avons déterminé l'efficience $\left(\mathrm{R}_{\max }\right)$ des dix souches, en modèle spermosphère, en deux expériences successives. L'analyse de variance à deux critères de classification (expérience et bactérie), pratiquée sur les données d'activité nitrogénasique, a montré que le facteur «bactérie» a eu un effet significatif ; en revanche, le facteur "expérience" et l'interaction de ces deux facteurs n'ont pas d'effet significatif (tableau IV). Les valeurs moyennes d'activité nitrogénasique des souches bactériennes ont varié entre 650 et
$\operatorname{Rmax}\left(\mathrm{C}_{2} \mathrm{H}_{4} /\right.$ tube/jour $)$

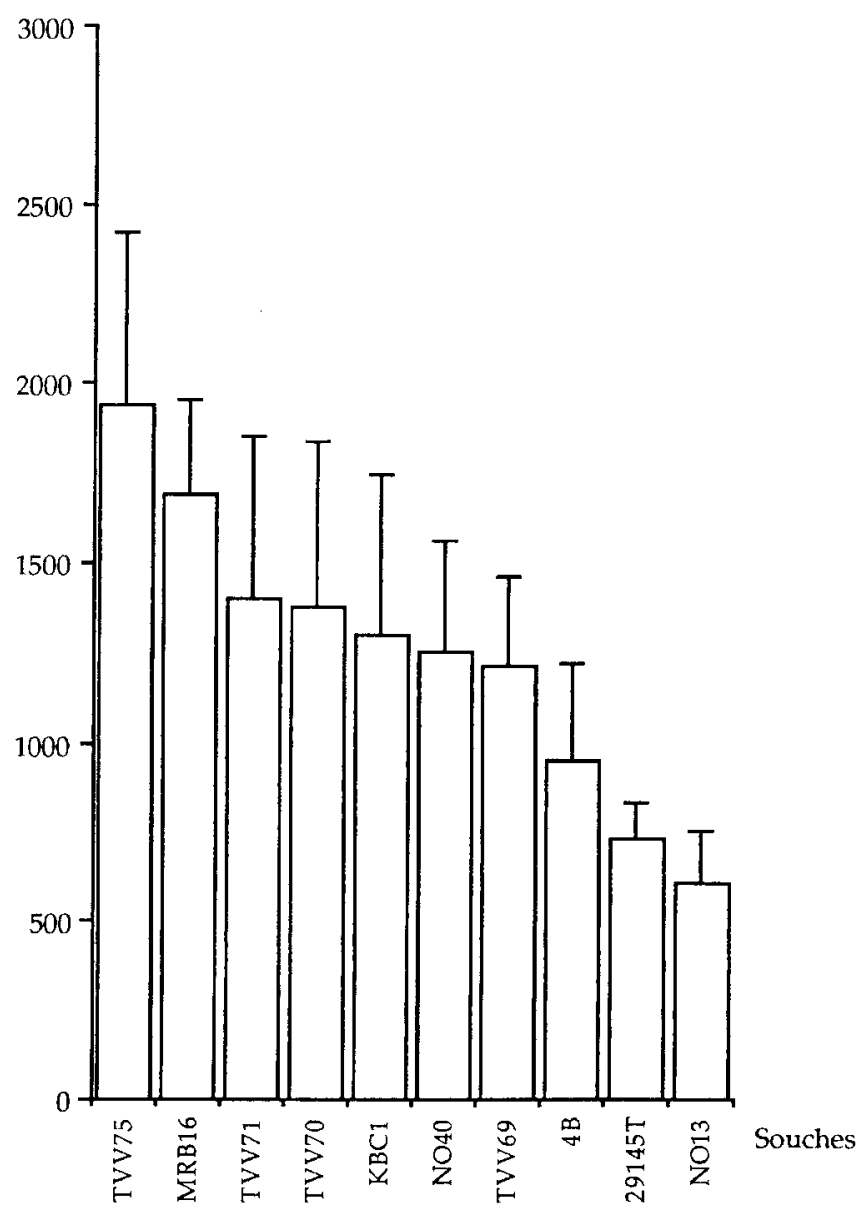

Fig 3. Comparaison en modèle spermosphère, de l'activité nitrogénastique des souches bactériennes isolées de la rhizosphère du riz d'origines variées : les quatre souches TVV sont des Burkholderia vietnamiensis isolées du sol de Bính Thanh, MRB16, KBC1, NO40, 4B, 29145 sont des Azospirillum isolés respectivement du Bangladesh, d'Irak, d'Égypte, de Camargue et du Brésil, NO13 est un Enterobacter cloacae isolé en Égypte. Le cultivar de riz Huyêt Ròng a été utilisé dans toutes les expériences. Les barres d'histogramme représentent la valeur moyenne de $R_{\text {max }}$ \pm intervalle de confiance à $95 \%$. 
Tableau IV. Résultats de l'analyse de variance de $\mathrm{R}_{\max }\left(\mathrm{nmol} \mathrm{C}_{2} \mathrm{H}_{4} /\right.$ tube/jour), mesuré en tubes spermosphère en fonction de la souche bactérienne inoculée et de l'expérience.

\begin{tabular}{lllllc}
\hline Source de variations & $D D L$ & Somme des carrés & Carrés moyens & $F$ & $P$ \\
Bactérie & & & & \\
Expérience & 9 & 14783153 & 1642572 & 5,86 & $<0,0000$ \\
Bactérie x expérience & 1 & 78587 & 78587 & 0,28 & 0,60 \\
Résidu & 9 & 675849,6 & 75094 & 8,27 & 0,98 \\
Total & 80 & 22436190 & 280452 & &
\end{tabular}

Le cultivar Huyêt Ròng a été utilisé dans ces expériences. Les tests $\mathrm{F}$ font intervenir le rapport entre le carré moyen des écarts du facteur étudié et le résidu.

1900 nmoles $\mathrm{C}_{2} \mathrm{H}_{4}$ /tube/jour) et sont rapportées dans la figure 3. La souche TVV75 fait partie des souches les plus efficientes.

Ce critère d'efficience a été utilisé pour sélectionner la souche TVV75, qui par ailleurs a montré d'autres propriétés intéressantes comme la prodution de sidérophores (Meyer et al, 1995) et l'inhibition de champignons pathogènes (Trân Van, 1994). Cette souche inoculée au riz dans des expériences en pots au Viêt-nam a permis d'augmenter les rendements de façon significative (Trân Van et al, 1994).

\section{DISCUSSION}

\section{Fixation d'azote associé au riz}

La mesure de l'Ara est une méthode simple, rapide et utilisable sur des plantes entières non perturbées. L'activité réductrice d'acétylène du riz cultivé sur le sol vietnamien de Bình Thanh atteint 360 nmoles de $\mathrm{C}_{2} \mathrm{H}_{4} \mathrm{~h}^{-1}$ plante ${ }^{-1}$ au huitième jour et montre qu'il existe un certain potentiel de fixation d'azote rhizosphérique dans les sols vietnamiens. Omar et al 1989 , dans les mêmes conditions de culture, ont observé un dégagement d'éthylène présentant aussi un pic au huitième jour, pour les sols de Giza et de Moshtohoor (Égypte), avec des valeurs respectives de 1300 et 2450 nmoles d'éthylène $\mathrm{h}^{-1}$ plante $^{-1}$, très supérieures à celles de cette étude. Ce pic correspond à l'effet spermosphère mis en évidence par Balandreau et al (1971) : il s'explique par l'épuisement des réserves de la graine. Sur un sol de Camargue, Hamad Fares
(1976) a mesuré une activité du même ordre que celle rapportée ici (500 nmoles d'éthylène $\mathrm{h}^{-1}$ plante $\left.{ }^{-1}\right)$. Des valeurs plus faibles ont été mesurées sur des sols de rizière du Bangladesh par Rahman et al (1996). Ces activités ont été mesurées sur des sols différents, mais aussi en présence de cultivars de riz très différents, ce qui rend difficile l'interprétation de leurs variations.

\section{Les populations de fixateurs d'azote dans la rhizosphère du riz}

II y avait 2,8 $\times 10^{8}$ fixateurs d'azote par gramme de sol, dans la rhizosphère du riz sur le sol de Bình Thanh. Ce chiffre est parmi les plus élevés de ceux que l'on trouve dans la littérature qui vont de $10^{4}$ à $10^{9}$ (Kobayashi et al, 1967 ; Nayak et al, 1986). La microflore totale cultivable représente $6,5 \times 10^{8}$ bactéries par gramme de sol rhizosphérique. En utilisant la même technique sur sol égyptien, Omar et al (1989) a dénombré une microflore totale cultivable de niveau similaire ( 7 à $8 \times 10^{8}$ bactéries/gramme de sol sec) alors que pour Rahman et al (1996) sur des sols du Bangladesh elle était moins dense (2 à $510^{8} \mathrm{bact} / \mathrm{g} \mathrm{sol} \mathrm{sec}$ ) (tableau V). Le dénombrement de la microflore totale cultivable sur milieu gélosé a permis de calculer que les fixateurs représentent environ $40 \%$ des bactéries totales cultivables, ce qui est très élevé, et témoigne d'une population fixatrice d'azote très développée dans notre système. En effet, mis à part le sol très fertile de Giza en Égypte ( $79 \%$ de fixateurs d'azote) tous les autres sols pour lesquels on possède des chiffres comparables ont des proportions de fixateurs 
Tableau V. Abondance de la microflore totale et des bactéries fixatrices d'azote dans différents sols selon divers auteurs.

\begin{tabular}{lllr}
\hline Origine des sols & $\begin{array}{l}\text { Microflore totale } \\
\text { cultivable }\end{array}$ & $\begin{array}{l}\text { Bactéries fixatrices } \\
\text { d'azote }\end{array}$ & \% fixateurs d'azote \\
\hline Giza (Égypte) & $71 \times 10^{7}$ & $56 \times 10^{7}$ & 79 \\
Moshtohoor (Égypte) & $82 \times 10^{7}$ & $23 \times 10^{7}$ & 28 \\
Brahmapoutre (Bangladesh) & $47 \times 10^{7}$ & $7 \times 10^{7}$ & 15 \\
Gange (Bangladesh) & $20 \times 10^{7}$ & $1,4 \times 10^{7}$ & 7 \\
Camargue (France) & $6 \times 10^{7}$ & $0,4 \times 10^{7}$ & 39 \\
Binh Thanh (Viêt-Nam) & $71 \times 10^{7}$ & $28 \times 10^{7}$ & \\
\hline
\end{tabular}

Pour les sols égyptiens, il s'agit des travaux d'Omar et al (1989); pour les sols du Bangladesh, des travaux de Rahman et al (1996). a Nombre de bactéries par gramme de matière sèche de sol rhizosphérique.

plus faibles : de 7 (Camargue, Bangladesh) à $28 \%$ (Moshtohoor, Égypte) (tableau V).

\section{Isolement et identification des bactéries fixatrices d'azote les plus nombreuses}

Balandreau (1983) a suggéré d'utiliser une source de carbone proche de celle utilisée par les bactéries dans la rhizosphère, c'est-à-dire les exsudats racinaires, comme milieu d'enrichissement sélectif pour l'isolement des bactéries rhizosphériques. Le modèle spermosphère permet d'isoler les bactéries adaptées à la plante en évitant les biais liés à l'emploi d'une source de carbone artificielle.

Les souches fixatrices d'azote isolées à partir de l'environnement et en particulier de la rhizosphère appartiennent à de très nombreuses espèces différentes et leur identification présente parfois des difficultés car beaucoup de ces bactéries n'ont pas fait l'objet d'étude taxonomique poussée (Balandreau, 1983 ; Ueda et al, 1995). Dans le sol sulfaté acide étudié, les bactéries fixatrices d'azote les plus abondantes dans la rhizosphère du riz ont été identifiées, de façon préliminaire, par le système API $20 \mathrm{NE}$ à l'espèce [Pseudomonas] cepacia. Cette espèce récemment rebaptisé Burkholderia cepacia par Yabuuchi et al (1992), n'étant pas connue pour contenir des diazotrophes, une étude polyphasique de la taxonomie des souches isolées a été engagée. Cette étude a montré qu'elles constituent une espèce nouvelle, $B$ vietnamiensis, très proche de $B$ cepacia (Gillis et al, 1995).
Le caractère nouveau de la microflore ainsi mise en évidence peut être lié aux caractères très particuliers des sols sulfatés acides étudiés, en particulier leur $\mathrm{pH}$ très bas. II ne faut cependant par oublier que la taxonomie incertaine du genre Burkholderia impose de revisiter des publications antérieures où ces bactéries auraient pu être décrites sous un autre nom : c'est ainsi qu'Asanuma et al (1980) ont déterminé comme $B$ cepacia l'espèce bactérienne dominante dans la rhizosphère des jeunes plants de riz qu'ils étudiaient au Japon ; il est possible qu'il s'agisse de $B$ vietnamiensis, mais en l'état de la taxonomie en 1980, ils ne pouvaient pas utiliser ce nom. Cette espèce représentait $60 \%$ de la microflore totale, les $40 \%$ restant étant des Bacillus sp. Ces auteurs n'ont pas envisagé qu'il s'agisse d'une espèce nouvelle : entre autres, ils n'ont pas testé ces souches pour la fixation d'azote. De même, Barraquio et Watanabe (1981) ont montré qu'aux Philippines la rhizosphère du riz était largement dominée par une bactérie proche, selon eux, du genre Pseudomonas ; ils n'ont jamais poussé plus loin la détermination et il serait intéressant de déterminer à nouveau ces souches à l'aide d'outils moléculaires (séquençage de I'ADN codant pour l'ARNr 16S). II n'est pas exclu qu'il s'agisse, en fait, de $B$ vietnamiensis.

\section{Influence du $\mathrm{pH}$}

Les souches de $B$ vietnamiensis ayant été isolées d'un sol acide dont le $\mathrm{pH}$ peut descendre jusqu'à 2, nous avons étudié l'effet $\mathrm{du} \mathrm{pH}$ sur 
l'activité réductrice d'acétylène d'une des souches de $B$ vietnamiensis précédemment isolées (TVV70) et nous l'avons comparée à celle de la souche d'Azosporillum irakense $\mathrm{KBC} 1$, elle-même connue pour réduire l'acétylène à pH 6 (Khammas et al, 1989). Les résultats obtenus ici montrent que la souche TVV70 isolée d'un sol acide est plus efficiente aux $\mathrm{pH}$ acides que la souche $\mathrm{KBC} 1$ isolée d'un sol au $\mathrm{pH}$ relativement alcalin $(\mathrm{pH} 7,7)$ qui est plus efficiente aux $\mathrm{pH}$ plus proches de la neutralité. Ces résultats sont en accord avec les observations de Khammas et al (1989) qui ont montré que l'activité nitrogénasique de la souche $\mathrm{KBC} 1$, sur milieu semi-solide est optimale à $\mathrm{pH}$ 6,5. Par ailleurs, Tabacchioni et al (1993) ont démontré une bonne colonisation et la persistance de $B$ vietnamiensis dans un sol calcaire à $\mathrm{pH} 8,8$. Cette espèce semble donc avoir une bonne capacité de survie dans une large gamme de $\mathrm{pH}$.

\section{Comparaison des souches}

Il est maintenant bien établi qu'il existe une interaction entre le cultivar d'une plante et la fixation d'azote qui lui est associée, et les différences dans la composition des exsudats pourraient expliquer ces variations (Loper et Schroth, 1986). Des travaux antérieurs ont déjà montré ces variations pour le riz (Ladha et al, 1987) ainsi que sur diverses céréales (Neal et al, 1973 ; Day et al, 1975 ; Ruschel et al, 1975 ; Von Bülow et Döbereiner, 1975). Pour le riz, il peut même exister des différences entre mutants végétaux (Rinaudo et Dommergues, 1971 ; Charyulu et al, 1985). C'est pourquoi les mesures d'efficience des souches fixatrices d'azote de la littérature sont difficiles à comparer entre elles.

Toutefois, les résultats présentés ici avec le cV Huyêt Ròng confirment ceux de Charyulu et al (1985) qui rapportaient une efficience élevée de la souche $4 \mathrm{~B}$ associée au cultivar Delta, ainsi que ceux de Heulin et al (1989) qui avaient montré l'avantage de la souche MRB16 sur la NO40 avec respectivement le cv Nizershail et le cv Giza 171.

Cette étude confirme aussi la grande variabilité d'efficience des souches d'Azospirillum pour la fixation d'azote que Khammas et al (1989) avaient déjà mesuré pour des souches
d'A irakense $(480$ à 1620 nmoles $\mathrm{C}_{2} \mathrm{H}_{4}$ /plante/jour).

Les souches de Burkholderia étudiées ici se sont révélées remarquablement efficientes (> 1200 nmoles $\mathrm{C}_{2} \mathrm{H}_{4}$ /plante/jour). La plus simple des hypothèses pouvant expliquer ceci est que l'équipement enzymatique des $B$ vietnamiensis est très fourni, et lui permet une croissance sur de très nombreux substrats (Gillis et al, 1995). Ces bactéries valoriseraient au mieux les composés divers exsudés par la plante : en cela, leur stratégie adaptative apparaît comme très différente de celle des Azospirillum, chez qui la gamme de substrats utilisables comme source de carbone est, au contraire, très réduite.

\section{CONCLUSION}

La présente étude a eu pour premier effet la mise en évidence de populations importantes d'une espèce bactérienne nouvelle, pour laquelle on a proposé, par ailleurs, le nom de $B$ vietnamiensis (Gillis et al, 1995).

Ceci confirme la grande diversité du peuplement microbien fixateur d'azote des racines de riz qui constitue, pour les biotechnologies une ressource insuffisamment exploitée : on trouve, en effet, dans cette microflore, une grande variété de propriétés métaboliques utiles. La capacité de fixer l'azote atmosphérique en est une, lorsque la croissance de la plante est limitée par la disponibilité de cet élément, ce qui est le cas le plus fréquent.

L'expérience acquise dans le cas de la riziculture égyptienne a montré qu'il était possible de sélectionner, parmi les fixateurs d'azote présents, une souche efficiente - un Azospirillum brasilense, en l'occurrence - qui a été utilisée pour inoculer le riz au champ : quand l'azote est le facteur limitant de la récolte, cette pratique assure une augmentation du rendement (Omar et al, 1992).

Ici cette approche et ces concepts ont été mis en cuvre dans le cas de la riziculture en sols sulfatés acides du Viêt-nam car les conditions pédoclimatiques étaient très différentes. Ceci explique sans doute, la présence et l'abondance d'un taxon inconnu jusqu'à présent. Cette approche a permis de montrer, ici encore, que les souches présentes spontanément différaient largement entre elles par leur capacité à fixer 
l'azote et qu'il était possible de sélectionner une souche particulièrement efficiente (TVV75).

La suite de la démonstration implique l'inoculation de cette souche dans des conditions de plus en plus proches des conditions de plein champ. L'inoculation en pots s'est révélée efficace et a produit des gains de récolte supérieurs à 20 \% (Trân Van et al, 1994). L'inoculation au champ a été testée avec le même succès, et ses résultats feront l'objet d'une publication ultérieure.

La stratégie d'inoculation du riz présentée ici apparaît dont foncièrement différente de celle qu'on utilise dans le cas du soja, par exemple : alors que chez les légumineuses l'inoculation a pour but d'introduire un symbionte qui est absent, elle a pour but, ici, d'utiliser la diversité existant spontanément dans le sol, pour y trouver un partenaire bactérien de la plante présentant une propriété intéressante (ici, une grande efficience pour la fixation d'azote) et d'inoculer cette souche afin de lui fournir un avantage sélectif par rapport aux autres souches lors de la colonisation des racines. Au total, on augmenterait l'abondance naturelle et incontrôlée d'un type bactérien utile afin de rendre significative son activité bénéfique sur la plante.

\section{REMERCIEMENTS}

Ce travail a été financé par le contrat $N^{\circ}$ STD 2A0119F de l'Union européenne ("Utilisation agronomique de l'inoculation bactérienne du riz: prédéveloppement"), dans le cadre d'un contrat de coopération entre le CNRS et l'Institut de biologie expérimentale d'Ho Chi Minh Ville. Nous tenons à remercier particulièrement $G$ Burtin, $J$ Rouiller, ainsi que B Gérard du CPB, pour leur analyse de sol.

\section{RÉFÉRENCES}

App AA, Watanabe I, Santiago-Ventura T, Bravo M, Daez-Jurey $C$ (1986) The effect of cultivated and wild rice varieties on the nitrogen balance of flooded soil. Soil Sci 141, 448-452

Asanuma S, Tanake H, Yatazawa M (1980) Pseudomonas cepacia - a characteristic rhizoplane microorganism in rice plants. Soil Sci Plant Nutrit 26, 71-78

Balandreau $J(1983)$ Microbiology of the association. Can J Microbiol 29, 8, 851-859

Balandreau J, Weinhard P, Rinaudo G, Dommergues $Y$ (1971) Influence de l'intensité de l'éclairement de la plante sur l'intensité de la fixation de l'azote dans sa rhizosphère. CEcol Plant 6, 341-351

Baldani JI, Baldani VLD, Seldin L, Döbereiner J (1986) Characterization of Herbaspirillum seropedicae gen nov, a root associated nitrogen-fixing bacterium. Int J Syst Bacteriol 36, 86-93

Bally R, Thomas-Bauzon D, Heulin T, Balandreau J, Richard C, De Ley J (1983) Determination of the most frequent $\mathrm{N}_{2}$-fixing bacteria in a rice rhizosphere. Can J Microbiol 29, 881-887

Bally R, Givaudan A, Bernillon J, Heulin T, Balandreau $J$, Bardin R (1990) Numerical taxonomic study of three $\mathrm{N}_{2}$-fixing yellow-pigmented bacteria related to Pseudomonas paucimobilis. Can J Microbiol 36, 850-855

Barraquio WL, Watanabe I (1981) Occurrence of aerobic nitrogen fixing bacteria in wetland and dryland plants. Soil Sci Plant Nutr 27, 1, 121-125

Berge O, Heulin T, Balandreau J (1991) Diversity of diazotroph populations in the rhizosphere of maize (Zea mays $\mathrm{L}$ ) growing on different French soils. Biol Fertil Soils 11, 210-215

Bulow JFW Von, Döbereiner J (1975) Potential for nitrogen fixation in maize genotypes in Brazil. Proc Natl Acad Sci USA 72, 2389-2393

Charyulu PBBN, Fourcassie F, Barbouche AK, Rondro Harisoa L, Omar AMN, Weinhard P, Marie R, Balandreau $J$ (1985) Field inoculation of rice using in vitro selected bacterial and plant genotypes. In : Azospirillum III: Genetics Physiology Ecology (W Klingmuller, ed), Springer-Verlag, Allemagne, 163179

Chistyakova IK, Kalininskaya TA (1984) Nitrogen fixation in takyr-like soils under rice. Microbiology 53, 101-105

Day JM, Neves MCP, Döbereiner J (1975) Nitrogenase activity on the roots of tropical forage grasses. Soil Biol Biochem 7, 107-112

Duong Duc T, Nguyên Thi H, Nguyên ML, Trân Van N, Vu Minh D, Nguyên Thi T (1987) Studies of the microbial nitrogen fixation in the rhizosphere of rice and rice paddies. Rapport pour la $2^{\mathrm{e}}$ réunion du réseau UNU (United Nations University) sur l'inoculation du riz, Bengkok, 1-6 février 1987

Gillis M, Trân Van V, Bardin R, Goor M, Hebbar P, Willems A, Segers $P$, Kerster $K$, Heulin $T$, Fernandez MP (1995) Polyphasic taxonomy in the genus Burkholderia leading to an amended description of the genus and the proposition of Burkholderia vietnamiensis $\mathrm{sp}$ nov for $\mathrm{N}_{2}$-fixing isolates from rice in Vietnam. Int J Syst Bacteriol 45, 274-289

Hamad-Fares I (1976) La fixation d'azote dans la rhizosphère du riz. Thèse doctorat d'État, université de Nancy-I

Heulin T, Guckert A, Balandreau J (1987) Stimulation of root exudation of rice seedlings by Azospirillum strains: carbon budget under gnotobiotic conditions. Biol Fertil Soils 4, 9-14 
Heulin T, Rahman M, Omar AMN, Rafidison Z, Pierrat $J C$, Balandreau J (1989) Experimental and mathematical procedures for comparing $\mathrm{N}_{2}$-fixing efficiencies of rhizosphere diazotrophs. J Microbiol Methods 9, 163-173

Heulin T, Berge O, Mavingui P, Gouzou L, Hebbar P, Balandreau J (1994) Bacillus polymyxa and Rahnella aquatilis, the dominant $\mathrm{N}_{2}$-fixing bacteria associated with wheat rhizosphere in French soils. Eur J Soil Bio/ 30, 1, 35-42

Khammas KM, Ageron E, Grimont PAD, Kaiser P (1989) Azospirillum irakense sp nov, a nitrogenfixing bacterium associated with rice roots and rhizosphere soil. Res Microbiol 140, 679-693

Khammas KM, Kaiser P (1992) Pectin decomposition and associated nitrogen fixation by mixed cultures of Azospirillum and Bacillus species. Can $J$ Microbiol 38, 794-797

Kobayashi M, Takahashi E, Kawaguchi K (1967) Distribution of nitrogen-fixing microorganisms in paddy soils of southeast asia. Soil Science 104, 113-118

Ladha JK, So RB, Watanabe I (1987) Composition of Azospirillum species associated with wetland rice plant grown in different soils. Plant Soil 102, 127 129

Lakshmi Kumari M, Kavimandan SK, Subba Rao NS (1976) Occurrence of nitrogen fixing spirillum in roots of rice, sorghum, maize and other plants. Indian J Exp Biol 14, 638-639

Loper JE, Schroth MN (1986) Importance of siderophores in microbial interactions in the rhizosphere. In : Iron, Siderophores and Plant Disease (TR Swinburne, ed), Plenum Press, New York, États-Unis, 85-98

Meyer JM, Trân Van V, Stintzi A, Berge O, Winkelmann $G$ (1995) Ornibactin production and transport properties in strains of Burkholderia vietnamiensis and Burkholderia cepacia (formerly Pseudomonas cepacia). BioMetals 8, 309-317

Nayak DN, Ladha JK, Watanabe I (1986) The fate of marker Azospirillum lipoferum inoculated into rice and its effect on growth, yield and $\mathrm{N}_{2}$ fixation of plants studied by acetylene reduction, ${ }^{15} \mathrm{~N}_{2}$ feeding and ${ }^{15} \mathrm{~N}$ dilution techniques. Biol Fertil Soils 2, 7-14

Neal JL, Larson RI, Atkinson TG (1973) Changes in rhizosphere populations of selected physiological groups of bacteria related to substitution of specific pairs of chromosomes in spring wheat. Plant Soil 39, 209-212

Omar AMN, Richard C, Weinhard P, Balandreau J (1989) Using the spermosphere model technique to describe the dominant nitrogen-fixing microflora associated with wetland rice in two Egyptian soils. Biol Fertil Soils 7, 158-163

Omar N, Berge O, Shalaan SN, Hubert JL, Heulin T, Balandreau J (1992) Inoculation of rice with Azospirillum brasilense in Egypt. Results of five different trials between 1985 and 1990 . Symbiosis $13,281-289$
Rahman M, Heulin T, Balandreau J (1996) Abundance and isolation of nitrogen-fixing bacteria from the major rice soils of Bangladesh. In : Biological Nitrogen Fixation Associated with Rice Production (M Rahman, ed), Kluwer Academic Publishers, UK, 171-179

Rasolomanana JL, Balandreau J (1987) Rôle de la rhizosphère dans la biodégradation de composés récalcitrants : cas d'une rizière polluée par des résidus pétroliers. Rev Ecol Biol Sol 24, 3, 443-457

Rinaudo G, Dommergues $Y$ (1971) Validité de l'estimation de la fixation biologique de l'azote dans la rhizosphère par la méthode de réduction de l'acétylène. Ann Inst Pasteur 121, 93-99

Roger PA, Ladha JK (1992) Biological $N_{2}$ fixation in wetland rice fields: Estimation and contribution to nitrogen balance. Plant Soil 141, 41-55

Roussos S, Garcia JL, Rinaudo G, Gauthier D (1980) Distribution de la microflore hétérotrophe aérobie et en particulier des bactéries dénitrifiantes et fixatrices d'azote libres dans la rhizosphère du riz. Ann Microbiol (Inst Pasteur) 131A, 197-207

Ruschel A, Henis Y, Salati E (1975) Nitrogen 15 tracing of $\mathrm{N}$ fixation with soil-grown sugar cane seedlings. Soil Biol Biochem 7, 181-182

Starkey RL, De PK (1939) A new species of Azotobacter. Soil Sci 47, 329-343

Tarrand JJ, Krieg NR, Döbereiner J (1978) A taxonomic study of the Spirillum lipoferum group, with descriptions of a new genus, Azospirillum gen nov and two species, Azospirillum lipoferum (Beijerinck) comb nov and Azospirillum brasilense sp nov. Can J Microbiol 24, 967-980

Tabacchioni S, Bevivino A, Chiarini I, Visca P, Del Gallo M (1993) Characteristics of two rhizosphere isolates of Pseudomonas cepacia and their potential plant-growth-promoting activity. Microb Releases 2, 161-168

Thomas-Bauzon D, Weinhard P, Villecourt P, Balandreau J (1982) The spermosphere model. I. Its use in growing, counting, and isolating $\mathrm{N}_{2}$-fixing bacteria from the rhizosphere of rice. Can $J$ Microbiol 28, 922-928

Trân Van V (1994) Burkholderia vietnamiensis sp nov, une protéobacterie fixatrice d'azote de la rhizosphère du riz isolée d'un sol sulfaté acide : taxonomie et effets de l'inoculation sur la croissance et le rendement du riz. Thèse d'université, université de Nancy-I, France, $360 \mathrm{p}$

Trân Van V, Mavingui $P$, Berge $O$, Balandreau J, Heulin T (1994) Promotion de croissance du riz inoculé par une bactérie fixatrice d'azote, Burkholderia vietnamiensis, isolée d'un sol sulfaté acide du Viêt-nam. agronomie 14, 697-707

Ueda T, Suga Y, Yahiro N, Matsuguchi T (1995) Remarkable $\mathrm{N}_{2}$-fixing bacterial diversity detected in rice roots by molecular evolutionary analysis of nifH gene sequences. J Bacteriol 177, 5, 1414-1417

Watanabe I, Barraquio WL (1979) Low levels of fixed nitrogen required for isolation of free-living $\mathrm{N}_{2}$-fixing 
organisms from rice roots. Nature (London) 277 , 565-566

Watanabe I, Lee KK (1977) Non-symbiotic nitrogen fixation in rice and rice fields. In: Biological Nitrogen Fixation in Farming Systems of the Tropics (A Ayanaba, PJ Dart, eds), Wiley and Sons, 289-305

Wetselaar R (1981) Nitrogen inputs and outputs of an unfertilized paddy field. In : Terrestrial Nitrogen Cycles. Ecol Bull Stockholm 33, 573-583

Yabuuchi E, Kosako Y, Oyaizu H, Yano I, Hotta H, Hashimoto Y, Ezaki T, Arakawa M (1992) Proposal of Burkholderia gen nov and transfer of seven species of the genus Pseudomonas homology group II to the new genus, with the type species Burkholderia cepacia (Palleroni and Holmes, 1981) comb nov. Microbiol Immunol 36, 1251-1275

Yoo ID, Fujii T, Sano Y, Komagata K, Yoneyama T, lyama $S$, Hirota $Y$ (1986) Dinitrogen fixation of riceKlebsiella associations. Crop Science 26, 297-301

Yuansheng $Q$, Shuping Z, Xiaozhen M, Dazi W, Junhua $H$ (1981) Study of nitrogen fixing bacteria associated with rice root. I. Isolation and identification of organisms. Acta Microbiologica Sinica 21, 472 